\title{
NLOS Identification for UWB Body Communications
}

\author{
Mohamed Tabaa ${ }^{1}$, Safa Saadaoui ${ }^{1}$, Mouhammad Chehaitly ${ }^{2}$, Abbas Dandache ${ }^{2}$ \\ ${ }^{1}$ Moroccan school of engineering science (EMSI), Research and Innovation Department, Casablanca, \\ Morocco \\ ${ }^{2}$ Laboratory of industrial engineering production and maintenance (LGIPM), Architecture of Embedded \\ System team (ASE), University of Lorraine, France
}

\begin{abstract}
In the last few years, a great attention has been paid to wireless communications for body area networks especially since the IEEE 802.15.6 standard. The main objective of this work is to present a good technique for identifying between both Line-Of-Sight (LOS) and Non-Line-Of-Sight (NLOS) propagation schemes for UWB both of both on-body and offbody communication. Our work is focalize in the first to extract the information using traditional features compared with our proposed methods and secondly to classify it using Support Vector Machine for objective to given a good recognition rate of identification between LOS and NLOS phenomena. This characterized was applied for UWB measurement by the antenna Electromagnetics Group (Body WiSeR).
\end{abstract}

\section{Keywords}

Ultra-wideband (UWB), Line-of-sight (LOS), Non-line-ofsight (NLOS), Stable distribution, Support Vector Machine (SVM), On-body, Off-body.

\section{INTRODUCTION}

The UWB systems consist to transmit a very short pulse of few nanoseconds over either a large frequency bandwidth from $500 \mathrm{MHz}$ to several $\mathrm{GHz}$, or a relative bandwidth larger than $20 \%$ of central frequency, according to the specification of the Federal Communication Commission (FCC). He represents hopeful technology for localization applications in harsh environments and critical applications in many domains $[1,2,4,13,14,18]$, notably for wireless personal area networks (WPANs) and especially for modern telemedicine systems using the IEEE 802.15.6 standard. The UWB technology is adapted to indoor localization thanks to a fine delay resolution and obstacle-penetration capabilities.

BANs have a great potential for UWB medicine systems and channel models have been standardized. Body Area Networks (BANs) have received a considerable attention in the last few years. With IEEE 802.15.6 standard, BANs use ultrawideband (UWB) in several domains like telemedicine, medical applications and communications for several situations On-body and Off-body.

A lot of challenges remain before implementation of UWB can be deployed on a large scale. These include signal acquisition, multi-user interferences, multipath and NLOS propagations $[1,2,5,8,16,19]$. The NLOS case is especially critical for most location-based applications because, in this case the propagation introduces positive bias in the estimation of distance, which can seriously affect the performance of localization. They are several methods to identify the NLOS phenomena, these methods can base in two scenarios: feature extraction and classification for identification. In the literature, they are several methods for extraction like traditional methods based on statistical and others methods like wavelet, PCA... The main objective of classification is identification, we are choosing for this research work SVM (Support Vector Machine) for objective identify between two classes LOS and NLOS.

In this paper, we propose a technique of identification for onbody and off-body communications. Our approach is based on stable distribution using SVM technique for objective to obtain a better identification. The measurements used were collected from a measurement campaign performed by Bodycentric Wireless Sensor Lab (Body WiSeR) with low loss coaxial cables to measure the transmission response. The rest of this paper is organized as follows. The proposed methods are presented in the section 2 . The section 3 is devoted to global discussion and results, before the conclusion.

\section{PROPOSED APPROACH}

In this section, we present our methodology for identification between LOS and NLOS phenomena for UWB on-body and off-body communications. Our work is focalized in extraction using several methods and identification using SVM classification for objectives given a good recognition rate. We begin by testing the data raw and compared with other strategy.

The first step consists in the feature extraction for goal to extract just only pertinent information, we are choosing in the first same traditional features and we are using others features: wavelet analysis and PCA. We describe our choice of method based on stable distribution for feature extraction and we are applied SVM for all feature for objective identification and giving the recognition rate. This identification helps in many domains like localization and mitigation, but the aim of this approach is to obtain a good rate of signal recognition with a better identification. In $[1,2,15,18]$, NLOS has been used for identification, localization and mitigation, with the same objectives in all these works: to find a method that facilitates the task for a good identification. In the literature, the NLOS conditions are presented by a signal more attenuated and that has smaller energy and amplitude; in LOS conditions, the signal is strong and presents high energy and amplitude. Generally, for body communications the information is presented by physiological signals, and the rate of such signals is much lower compared with other applications of UWB [14,16,17,18]. Fig. 1 and fig. 2 shows an obvious difference between LOS and NLOS situations for on-body and off-body communications. 

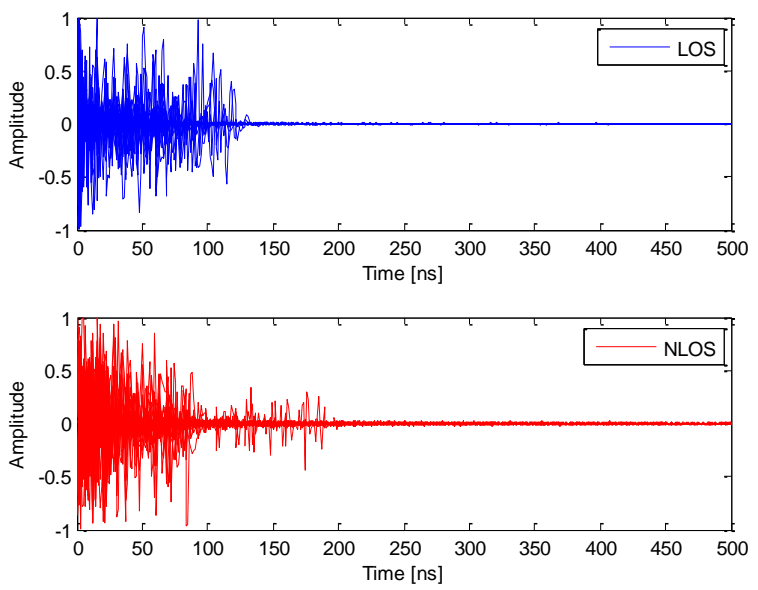

Figure 1: Difference between LOS and NLOS for on-body communications
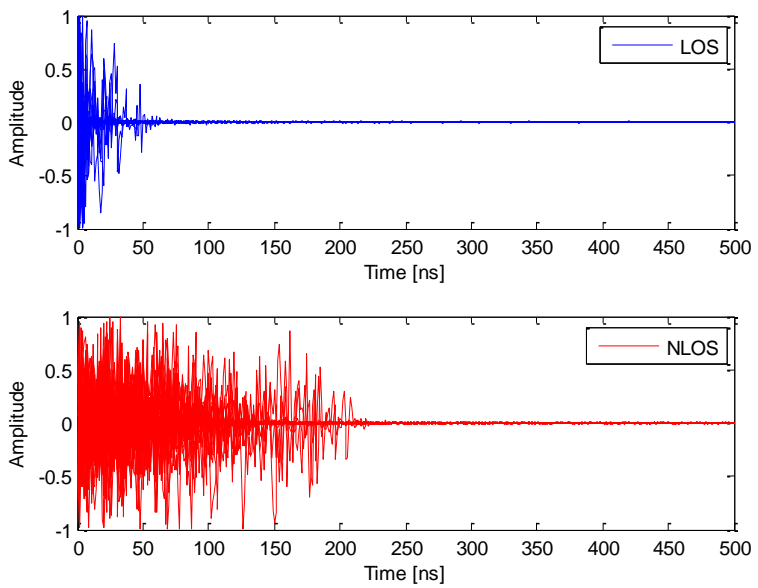

Figure 2: Difference between LOS and NLOS for off-body communications

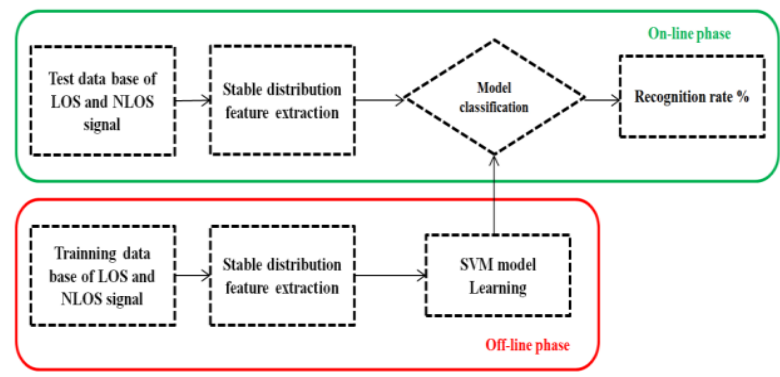

Figure 3: Proposed approach

\subsection{Traditional features}

In this part, we describe the statistical features used in literature and that the need to distinguish between the different classes LOS and NLOS phenomena. In [1,2,15,18], basic traditional statistical methods are used for feature extraction to prove the choice of others methods. With the same idea, we are using some statistical methods compared with our own but for medical applications in two situations On-body and Off-body. The selections features we will consider are as follows:
1) Energy of the received signal:

$$
f(x)=\int_{-\infty}^{+\infty}|r(t)|^{2} d t
$$

2) Mean excess delay:

$$
\tau_{M E D}=\int_{-\infty}^{+\infty} t \varphi(t) d t
$$

Where $\varphi(t)=|r(t)|^{2} / \varepsilon_{y}$

3) RMS delay spread:

$$
\tau_{\text {RMS }}=\int_{-\infty}^{+\infty}\left(t-\tau_{M E D}\right)^{2} \varphi(t) d t
$$

4) Kurtosis:

$$
k=\frac{1}{\sigma_{|r|} T} \int_{T} r(t)-\mu_{|r|}[] d t
$$

Where $\mu_{|r|}=\frac{1}{T} \int_{T}|r(t)| d t$ and $\sigma^{2}|r|=\frac{1}{T} \int_{T}\left[\left|r(t)-\mu_{|r|}\right|\right]^{2} d t$

5) Entropy:

$$
H(X)=E[I(X)]=E[-\ln (P(X))]
$$

Where $\mathrm{E}$ is the expected value operator, and $I$ is the information content of $X$.

6) Variance:

$$
\operatorname{Var}(x)=\int x^{2} f(x) d x-\mu
$$

\subsection{Wavelet}

The wavelet function has to have a compact support [19], that is its average value in time domain must be zero $\int_{-\infty}^{+\infty} \Psi(t)=0$ and it has a finite energy $\int_{-=\infty}^{+m}|\Psi(t)|^{2} d t<\infty$

Hence, a signal at higher resolution level $i+1$ can be separated into two signals, approximation and details, at the lower resolution level $i$ :

$$
A_{i+1} f=A_{i} f+D_{i} f=\sum_{n} a_{i n} \varphi_{i n n}+\sum_{n} d_{i n n} \Psi_{i n}
$$

In the discrete wavelet transform, the approximation signal $A_{i} f$ is further decomposed onto two subspaces of approximation and details. If the decomposition is repeated until the approximation becomes zero, the signal can be expressed as a composition of wavelet functions at different scales:

$$
f(x)=\sum_{i_{n} n} d_{i_{\infty} n} \Psi_{i_{m} n}(x)
$$

\subsection{Principal component analysis}

Principal Component Analysis (PCA) is a well-known linear method for feature extraction and dimensionality reduction; it reduces the redundancy by calculating the eigenvectors of the covariance matrix of the input [20]. The PCA is for objective only allows linear dimensionality reduction. If the data have more complicated that cannot reduced by traditional PCA, but in our case the data is only physiological signal and in this context the traditional PCA can help to simplify data.

The linear PCA is performed in the mapped space whose dimension is assumed to be larger than the number of training samples:

$$
x_{i} \rightarrow \Phi\left(x_{i}\right)
$$

The PCA can be computed such that the vectors $\Phi\left(x_{\mathrm{i}}\right)$ appear only within scalar products. 


\subsection{SVM Classifier}

The support vector machine (SVM) is based on a simple idea that originated in statistical learning theory by Vapnik [5]. This simplicity comes from the fact that this technique uses a simple linear method, but applied in high-dimensional feature space non-linearly related to the input space. It represents one of the most broadly used classification techniques because of its robustness, its performance and its rigorous underpinning compared to other techniques like neural networks.

For the identification, support vector machines separate the different classes of data by a hyper plane [ ]

$$
(w, \varphi(x))+b=0
$$

Corresponding to the function

$$
F(x)=\operatorname{sign}((w, \varphi(x))+b)=0
$$

Where $\varphi(\mathrm{x}) \mathrm{F}(\mathrm{x})$ is a predetermined function, and $w$ and $b$ are unknown parameters of the classifier.

These parameters are determined based on the training set $\left\{X_{k} l_{k}\right\}_{k=1}^{N}$, where $X_{k} \in R^{n}$ and $l_{k} \in\{-1,+1\}$ are the inputs and labels, respectively. In some cases, the two classes can be separated and the SVM determines the separating hyper plane that maximizes the margin between the two classes. Generally, most practical problems involve classes that are not separable. In this case, the SVM is obtained by solving the following optimization problem:

$$
\operatorname{argmin}_{1 b_{b}} \frac{1}{2}\|w\|^{2}+\gamma \sum_{k=1}^{N} \varepsilon_{k} \text { with } l_{k y}\left(X_{k}\right) \geq 1-\varepsilon_{k}, \forall k
$$

Where $\varepsilon_{\mathrm{k}}$ are slack variables that allow the SVM to tolerate misclassifications and $\gamma$ controls the trade-off between minimizing training errors and complexity [5].

\section{SIMULATIONS AND RESULTS}

\subsection{Data presentation}

With the aim to study the characteristics of UWB, we worked with the data collected by the Antenna \& Electromagnetics Group (Body WiSeR). All the parameters for the measurement are presented in Table 1. All the measurements were collected in a room that is $3 \mathrm{~m}$ high and which geometry is described in Fig. 4. More information can be found in [16]

Table 1: Parameters of Body WiSeR database

\begin{tabular}{|c|c|}
\hline Parameters & Values \\
\hline Frequency range & $3-10 \mathrm{GHz}$ \\
Frequency sampling & $4,37 \mathrm{MHz}$ \\
Maximum time delay & $228.8 \mathrm{~ns}$ \\
Maximum observable distance & $68.6 \mathrm{~m}$ \\
Frequency span & $7 \mathrm{GHz}$ \\
Maximum temporal resolution & $0.14 \mathrm{~ns}$ \\
Maximum spatial resolution & $43 \mathrm{~mm}$ \\
Time bin size & $0.14 \mathrm{~ns}$ \\
Transmit power & $0 \mathrm{dBm}$ \\
IF bandwidth & $3 \mathrm{KHz} / 101 \mathrm{dBm}$ \\
\hline
\end{tabular}

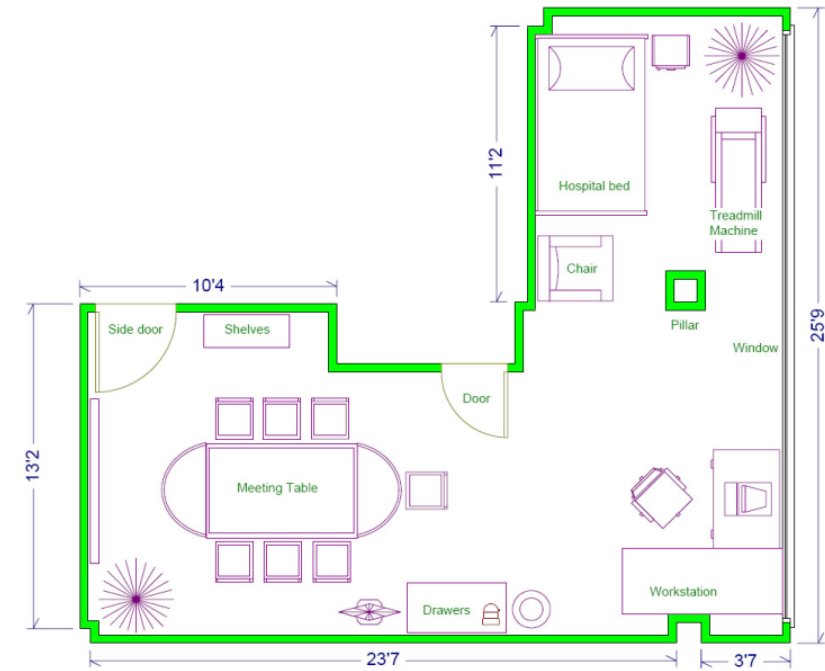

Figure 4: Dimensions and geometry of the Body Centric Wireless Sensor Lab where the indoor radio propagation measurements are performed. The sensor lab height is $\mathbf{3} \mathbf{~ m}$

\subsection{Extraction and classification results}

The strategy for identification between LOS and NLOS phenomena will be described below. In the present work, all data are obtained from Body WiSeR laboratory measurements as described in [16]. The measurements are based on the scenarios for on-body and off-body communications. In both situation, the LOS scenarios are presented in 1 to 56 sensors implemented in the front of body and the NLOS are presented in 57 to 110 sensors in the back as depicted in Fig. 5.

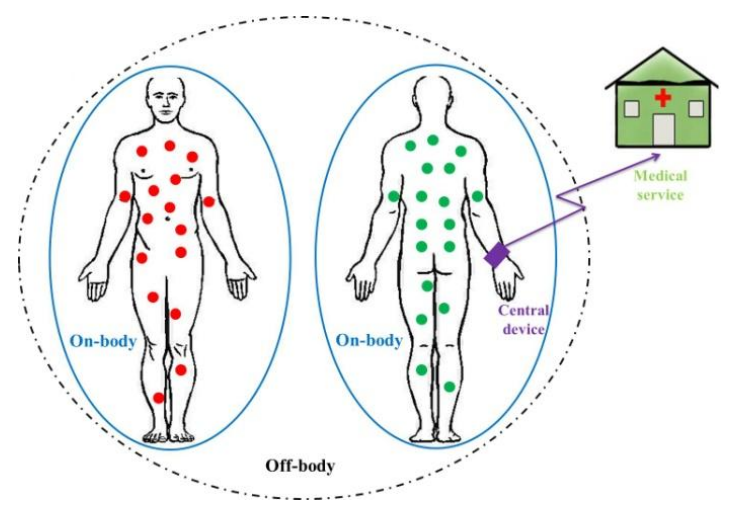

Figure 5: On-body index locations for NLOS and LOS

LOS and NLOS signals are grouped in a matrix to facilitate the data processing. In body communication data are present by physiological signals and the big difference between two cases requires separating between two phenomena LOS and NLOS. To identify LOS and NLOS signals for On-body and Off-body cases, several parameters can be analyzed: the impulse response (maximum amplitude, energy), frequency response (fading), power delay profile $P D P$, rise time, root mean square $R M S \ldots$. Some of these parameters will be held for the simulations. Figures 1 and 2 show a clear difference between LOS and NLOS signals for both On-body and Offbody cases. The first arrivals paths are strongest for LOS Offbody signals than LOS On-body. NLOS signals are much dispersive in Off body case and those for On body are much attenuated. This is due to On-Body propagation medium where signals are more affected by absorption and reflection phenomena. Figure 6 shows the energy distribution for Onbody and Off-body schemes. 

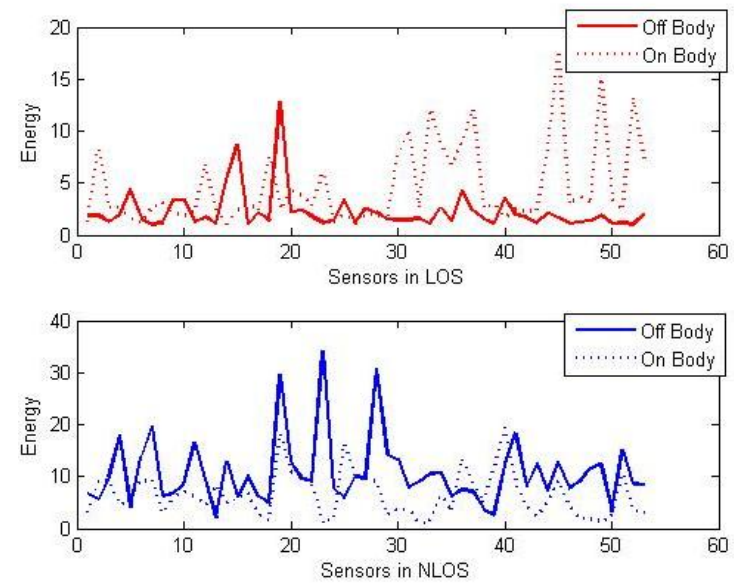

Figure 6: On-body and Off-body energy for LOS and NLOS signals
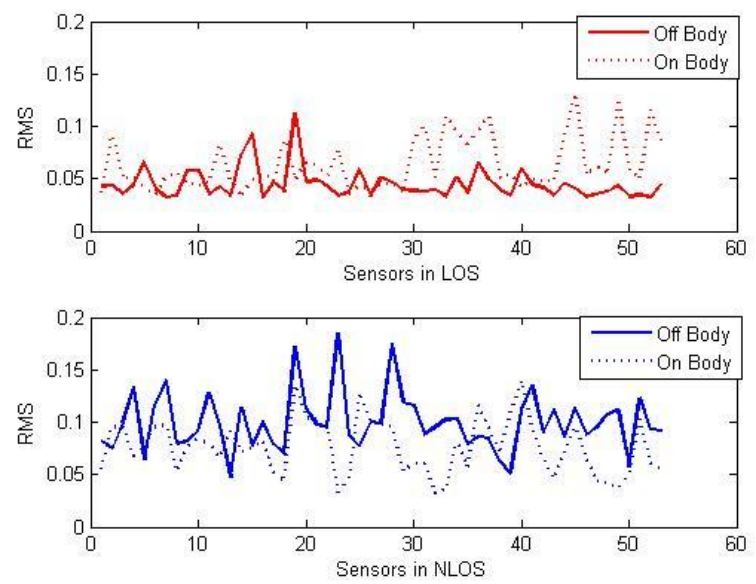

Figure 7: On-body and Off-body RMS for LOS and NLOS signals

Strong energy for NLOS signal in Off-Body scheme. This signal results from the sum of a plurality of reflected signals. In On-body case, LOS signal possess a strong energy. The temporal dispersion of the signal's energy which is measured by RMS is larger for NLOS On-body and Off-body signals as depicted in Figure 7.

The amplitude, energy and RMS will influence the impulse response as well as frequency response and PDP for all scenarios. Fig 8,9,10 and 11 show the difference between Onbody and Off-body frequency response and power delay profile. Once signals identification is made, a classification of LOS and NLOS data based on SVM method will be held. The first scenario uses data raw extraction method which showed $50 \%$ recognition rate between LOS and NLOS signals. In the second scenario, we extract only some information from data using traditional feature extraction described in section II. We use kurtosis, mean, entropy, RMS, energy and variance to extract only the pertinent information and we use the SVM classifier. In this case, we still get the same recognition rate of $50 \%$. In third scenarios, we extract the pertinent information using two techniques wavelet and PCA. After having reduced the size of data, we applied SVM classification. Both of wavelet and PCA gives only $50 \%$ of recognition rate. In the fourth scenario, we extract the pertinent information from data using stable distribution. After having reduced the size, we proceed to the identification using SVM. The results are shown in Table 2.

Table 2: All results of extraction and classification scenarios for different identification methods in both onbody and off-body context

\begin{tabular}{|c|c|c|}
\hline $\begin{array}{c}\text { Feature extraction } \\
\text { On-body and off-body }\end{array}$ & Identification & $\begin{array}{c}\text { Recognition } \\
\text { rate }\end{array}$ \\
\hline Data raw & SVM & $50 \%$ \\
\hline $\begin{array}{c}\text { RMS, mean, entropy, } \\
\text { variance }\end{array}$ & SVM & $40.38 \%$ \\
\hline $\begin{array}{c}\text { Kurtosis, mean, entropy, } \\
\text { variance }\end{array}$ & SVM & $50 \%$ \\
\hline RMS, mean, entropy, energy & SVM & $50 \%$ \\
\hline $\begin{array}{c}\text { RMS, Variance, kurtosis } \\
\text { energy }\end{array}$ & SVM & $53.84 \%$ \\
\hline DWT & SVM & $50 \%$ \\
\hline PCA & SVM & $50 \%$ \\
\hline Stable distribution & SVM & $87.5 \%$ \\
\hline
\end{tabular}
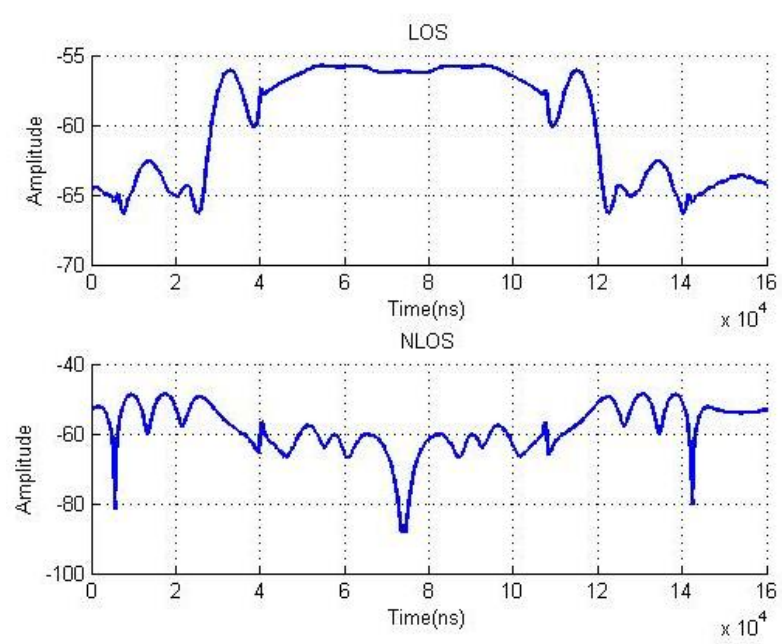

Figure 8: On-body frequency response for NLOS and LOS
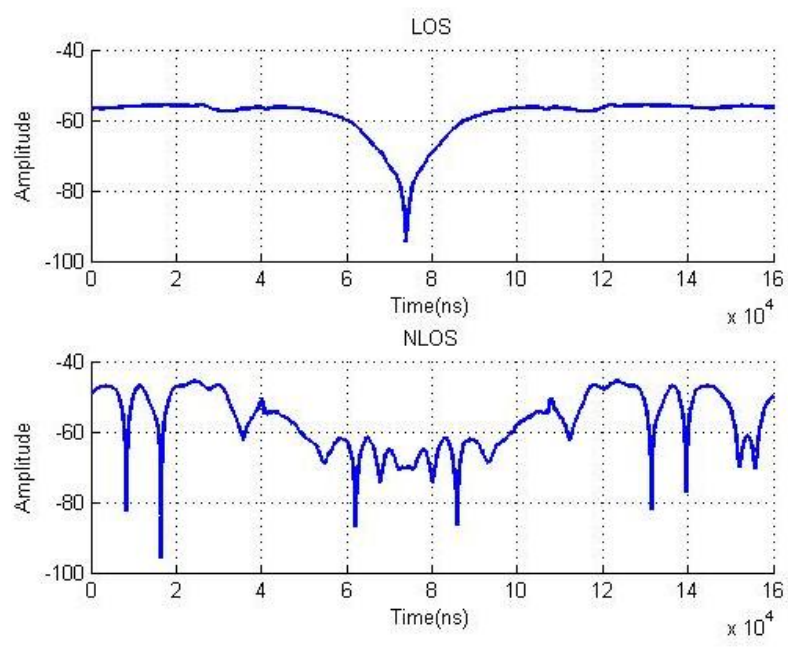

Figure 9: Off-body frequency response for NLOS and LOS 

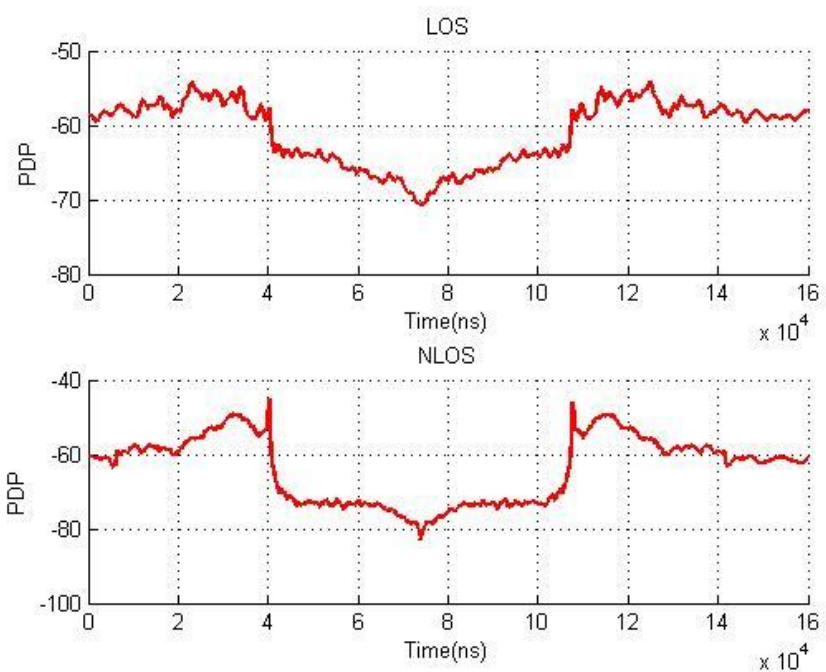

Figure 10: On-body power delay profile for NLOS and LOS
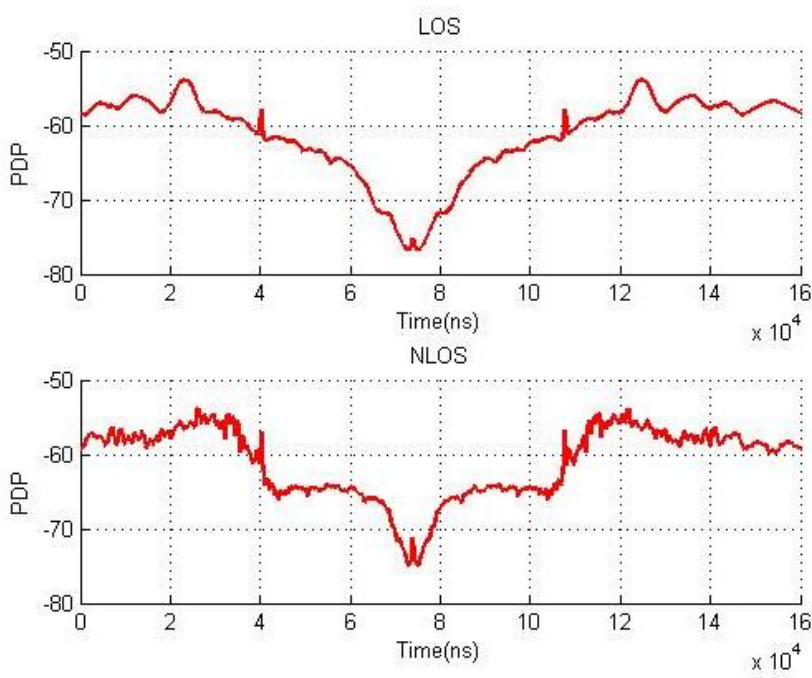

Figure 11: Off-body power delay profile for NLOS and LOS

\section{CONCLUSION}

In this paper we described a methodology of identification between LOS and NLOS phenomena in both of On-body and Off-body situation. We are using for that two strategy's feature extraction based on stable distribution and classification for identification using SVM. This approach gives good results with $87.5 \%$ of recognition rate compared to traditional extraction, wavelet and PCA. By using both the stable distribution and the SVM classifier we developed a technique that is capable of distinguishing two critical LOS and NLOS phenomena for on and off body communications.

\section{ACKNOWLEDGMENT}

Special thanks for Dr. Rachid Saadane form EHTP Casablanca, Morocco for his cooperativeness and valuable help.

\section{REFERENCES}

[1] Stefano Marano, Wesley M. Gifford, Henk Wymeersch, Moe Z. Win, "Nonparametric Obstruction Detection for UWB Localization", Global Telecommunications Conference, GLOBECOM 2009, IEEE, 2009, 1-6.

[2] Stefano Marano, Wesley M. Gifford, Henk Wymeersch, Moe Z. Win, "NLOS Identification and Mitigation for Localization Based on UWB Experimental Data", IEEE Journal on Selected Areas in Communications, vol. 28, Issue 7, September 2010.

[3] Z. Mohammadi, R. Saadane, D. Aboutajdine, "Ultra Wide-Band Channel Characterization Using Generalized Gamma Distributions", Image and Signal Processing, vol.7340, pp.175-182, Springer 2012.

[4] J. Zhang, Z. Sahinoglu, P. Kinney, "UWB Systems for Wireless Sensor Networks", Proceedings of the IEEE "Invited Paper", vol. 97, no.2, Feb. 2009.

[5] C. Cortes, V. Vapnik, "Support Vector Networks", Machine Learning vol. 20, no.3, pp.273-297, 1995.

[6] Ming-Chang Lee, Chang To, "Comparison of Support Vector Machine and Back Propagation Neural Network in Evaluating the Enterprise Financial Distress", International Journal of Artificial Intelligence \& Applications (IJAIA), vol.1, no.3, July 2010.

[7] A. Karatzoglou, D. Meyer and K. Hornik "Support Vector Machine in R", Journal of Statistical Software, vol.15, no.9, Apr. 2006.

[8] S. Venkatesh, R. M. Buehrer, "Non-Line-of-Sight Identification in Ultra-Wideband Systems Based on Received Signal Statistics”, Antennas Propag., vol.1, no.6, pp.1120-1130, 2007

[9] Raffaele Di Bari, Qammer H. Abbasi, Akram Alomainyans, Yang Hao, "An Advanced UWB Channel Model for Body-Centric Wireless Networks", Progress in Electromagnetics Research, vol.136, pp.79-99, 2013.

[10] H. El Ghannudi, L. Clavier, N. Azzaoui, F. Septier, PA. Rolland, "Alpha-stable Interference Modeling and Cauchy Receiver for an IR-UWB ad hoc Network", IEEE Transactions on Communications, vol.58, issue 6, June 2010.

[11] J. H. McCulloch, "Simple Consistent Estimators of Stable Distribution Parameters", Communications in Statistics - Simulation and Computation, vol.15, issue 4, pp.1109-1136, 1986.

[12] W. Suwansantisuk, M. Z. Win, "Multipath Aided Rapid Acquisition: Optimal Search Strategies," IEEE Transactions on Information Theory, vol.53, no.1, pp.174-193, Jan. 2007.

[13] M. Z. Win, G. Chrisikos， A. F. Molisch，"Wideband Diversity in Multipath Channels with Nonuniform Power Dispersion Profiles", IEEE Transactions on Wireless Communications, vol.5, no.5, pp.1014-1022, May 2006.

[14] J. Khodjaev, Y. Park, A. S. Malik, "Survey of NLOS Identification and Error Mitigation Problems in UWB- 
Based Positioning Algorithms for Dense Environments," Annals of Telecommunications, June 2010, vol.65, issue 5-6, pp.301-311, Springer 2009.

[15] M. Tabaa, C. Diou, M. El Aroussi, B. Chouri, A. Dandache, "LOS and NLOS Identification Based on UWB Stable Distribution", IEEE International Conference on Microelectronics, December 15-18, Beyrouth, Lebanon, 10.1109/ICM.2013.6734961.

[16] Mohammad Monirujjaman Khan, Qammer H. Abbasi, Akram Alomainy, Yang Hao, "Performance of Ultra wideband Wireless Tags for On-Body Radio Channel Characterisation", International Journal of Antennas and Propagation, vol.2012, article ID 232564, 10 pages.

[17] Min Chen, Sergio Gonzalez, AthanasiosVasilakos, Huasong Cao, Victor C.M.Leung, "Body Area Networks: A survey", Journal of Special Issues on Mobility of
Systems Users, Data and Computing, vol.16, no.2, 2010, ISSN 1383-469X.

[18] Mohamed Tabaa, Camille Diou, Rachid Saadane, Abbas Dandache, «LOS /NLOS Identification based on stable distribution feature extraction and SVM classifier for UWB On-body communications », Procedia Computer Science Volume 32, pages 882-887, 2014.

[19] S. Mallat. A theory for multiresolution signal decomposition: The wavelet representation.IEEE Transactions on Pattern Analysis and Machine Intelligence, 11(7):674-693, July 1989.

[20] V.Savic, E.G.Larsson, J.Ferrer-Coll, P.Stenumgaard "Kernel Principal Component Analysis for UWB-Based Ranging", IEEE international workshop on signal processing advances in wireless communications, Toronto, 22-25 June 2014. 